\title{
Cómo el propio consumo de drogas de los mediadores recreativos tiene implicaciones preventivas
}

\author{
Amador Calafat*; Cesáreo Fernández*; Montse Juan****; Elisardo Becoña*, *** \\ * Irefrea \\ ** Universidad de Alicante \\ *** Universidad de Santiago de Compostela \\ Enviar correspondencia a: \\ Amador Calafat. Irefrea. Rambla, 15, 2 $3^{\mathrm{a}} .07003$ Palma de Mallorca. irefrea@irefrea.org. www.irefrea.org
}

Recibido: 15 de Octubre de 2004 Aceptado: 10 de Febrero de 2005.

\section{RESUMEN}

Se estudia la influencia que tiene el consumo de alcohol y otras drogas entre profesionales relacionados con el contexto recreativo de fin de semana sobre las actitudes y opiniones sobre las drogas. La muestra incluye 246 profesionales de cinco autonomías españolas que pertenecen a 4 sectores: 1 ) industria recreativa $(24,8 \%), 2)$ medios de comunicación expresamente dirigida a los jóvenes $(19,1 \%)$, 3) servicios sociales y programas preventivos $(44,3 \%)$ y 4 ) Responsables de asociaciones juveniles $(11,8 \%)$. La mayor parte son jóvenes y con estudios universitarios.

La mayoría (62\%) de estos mediadores se embriaga de vez en cuando, el $38,5 \%$ son consumidores por lo menos ocasionales de cannabis y el $25 \%$ consumen otras drogas ilegales al menos ocasionalmente. Los miembros de los servicios sociales y de las asociaciones juveniles consumen estas drogas con menor probabilidad que los agentes de la industria recreativa y de los medios de comunicación. La percepción de la peligrosidad del consumo de drogas es significativamente menor entre los que consumen.

Las creencias de estos profesionales sobre cuestiones importantes para la prevención no se adaptan en ocasiones a la evidencia científica (un 38,4\% de todos estos profesionales piensa que consumir cannabis no es perjudicial, porcentaje que pasa a un 58,2\% entre los que consumen cannabis).

Es importante que los programas preventivos tengan en cuenta la formación de los profesionales que trabajan en conexión inmediata con los jóvenes que salen a divertirse abordando en esta formación el tema de los propios consumos.

Palabras clave: uso de drogas recreativas, prevención, mediadores sociales, profesionales prevención, creencias.

\section{ABSTRACT}

We studied the influence of alcohol consumption and other drug use by professionals related to the weekend recreational context on their attitudes and opinions on drugs. The sample includes 246 professionals in five Spanish regions from four sectors: 1) recreational industry $(24.8 \%), 2$ ) the media expressly targeting the young $(19.1 \%), 3)$ social services and preventive programmes $(44.3 \%)$, and 4) juvenile association leaders $(11.8 \%)$. The majority are young with a university background.

The majority $(62 \%)$ of these professionals are inebriated from time to time, $38.5 \%$ are users, at least occasionally, of cannabis and $25 \%$ use other illegal drugs at least occasionally. There is a lower probability of those from the social services and juvenile associations using these drugs in comparison with those in the media. The perception of the danger inherent in drug use is significantly less among those who use.

On occasion, the beliefs of these professionals on important issues for prevention are not in line with scientific evidence (38.4\% of all these professionals believe that cannabis is not harmful and this percentage rises to $58.2 \%$ among those who do use cannabis.).

It is important that preventive programmes take into consideration the training of the professionals who work in the immediate environment of the young in their search for enjoyment their own uses, should be approached during this training.

Key words: Recreational drug use, prevention, social mediators, prevention professionals, beliefs.

\section{INTRODUCCIÓN}

a familia o los profesionales de la educación son colectivos tradicionalmente muy próximos a los jóvenes, con notable influencia sobre su socialización; pero en las sociedades modernas han ido cobrando importancia otros profesionales y colectivos por su especial relación con los jóvenes. Nos referimos a los profesionales del ámbito recreativo y de los medios de comunicación, los que trabajan en 
publicidad, los que crean o difunden elementos de consumo (indumentaria, moda, vehículos, complementos estéticos, música, etc.). La llegada de estos nuevos colectivos es el resultado de la aparición de nuevos espacios socializadores además del hogar y la escuela. Los jóvenes también se educan en los espacios recreativos de fin de semana, en los comercios creados para los jóvenes, las plazas donde los jóvenes pasan su tiempo libre, etc. Tenemos que tener en cuenta además los nuevos formatos de comunicación de los jóvenes como internet, los chats, los teléfonos móviles con sus logos, nuevas formatos para escuchar música, etc. Existe pues una heterogeneidad de instancias que crean las nuevas condiciones para la socialización de los jóvenes. Es por ello necesario conocer más estos contextos y estos profesionales para saber como funcionan y ejercen su influencia sobre los jóvenes pues seguro que ello tiene importancia en la etiología y la prevención del consumo de drogas.

El aumento del tiempo de ocio para grupos amplios de población ha sido en las sociedades industriales occidentales el resultado de una larga conquista social. Los jóvenes se han incorporado plenamente a esta situación y para ellos la diversión de fin de semana es un espacio privilegiado que ellos viven como algo plenamente suyo -en contraposición al resto de la semana (estudio o trabajo) que es el mundo que comparten con los adultos- y con gran intensidad. En este sentido el 30,5\% de los estudiantes españoles de 14 a 18 años declaran salir por las noches todos los fines de semana del último año'.

Alrededor de la 'movida' y del 'salir de marcha' se ha desarrollado una floreciente industria muy especializada que obviamente lucha para expandir su mercado. Las sociedades deben estar atentas a estos fenómenos ${ }^{2}$ pues a través del vínculo con ideales como la diversión, el placer, la felicidad, se puede desarrollar eficientes instrumentos de control social (recordemos la novela de Aldous Huxley “Un mundo feliz"). Estas potentes industrias y las nuevas tecnologías de la diversión pueden comprometer el grado de autonomía que tienen los jóvenes, adolescentes y niños para organizar y decidir su conducta frente a la diversión. El problema es que no sabemos demasiado de qué forma estas nuevas condiciones de socialización que representan los contextos recreativos influyen a los jóvenes y de qué forma pueden condicionar, entre otras cosas, el consumo de drogas. Esta dinámica está hoy básicamente en manos de la industria, a la que se está dejando la posibilidad no únicamente de que ofrezca unos servicios sino de definir los ideales y las prácticas concretas de lo que debe ser la diversión de los jóvenes, un aspecto crucial de la socialización.
En estos espacios recreativos encontramos distintos profesionales y mediadores sociales que cumplen distintas funciones, y de los que sabemos poco sobre sus conductas, como es su propio consumo de drogas, y en qué forma pueden influir en la forma de entender la diversión los jóvenes, incluido su consumo de drogas. Consideramos por ello que conocer mejor y colaborar con estos profesionales debería ser una area prioritaria para la prevención, puesto que ocupan lugares clave dentro de las nuevas formas de socialización. Dentro de esta orientación Shields ${ }^{3}$ remarca, tras un importante trabajo cualitativo con distintos profesionales de Hollywood (escritores, actores, directores, productores, ejecutivos y otros), que las normas y valores de personas conocidas de la industria del espectáculo se transmiten y condicionan la percepción y la actitud ante el consumo de drogas de la sociedad. Otro de los elementos puede ser los profesionales que crean la moda, que es un elemento de gran importancia para la toma de decisiones de los jóvenes, hasta el punto que buena parte de ellos afirman consumir drogas ilegales porque están de moda 4 .

Sin embargo los estudios sobre factores de riesgo se orientan más hacia los determinantes de tipo intrapersonal (genéticos, constitucionales, psicológicos) e interpersonal (familiares, amigos, escuela) y se están estudiando menos los factores ambientales ${ }^{5}$. Los factores relativos a la comunidad incluidos en estos modelos y teorías se refieren frecuentemente a la disponibilidad de sustancias, la deprivación socioeconómica y la existencia de normas tolerantes hacia el consumo dentro de la comunidad. En los últimos quince años se ha ido impulsando el estudio de las interacciones entre el individuo y su entorno social y físico como determinantes del uso y abuso de drogas $^{6}$. Sin embargo, la aplicación de este campo de estudio de las interacciones individuo con los espacios recreativos es aún muy escasa. Además existe controversia acerca del significado que adquieren las drogas en el ámbito recreativo, e incluso algunos investigadores minimizan los problemas que puedan producir, ${ }^{7,}$, mientras que otros son más críticos ${ }^{9,10} \sin$ que exista acuerdo sobre el papel que juegan según se plantea en diversos estudios que tratan de mirar las condiciones culturales ${ }^{11,12,13,14}$.

Pero, independientemente del papel que cumplan las drogas en estos ambientes -que por descontado es muy complejo-, lo que nadie niega es la presencia frecuente y la profunda interacción que se establece entre las drogas y lo recreativo. "Músicas como el trance y el house, típicas del Reino Unido y de Europa occidental, no sonarían como lo hacen si no estuvieran diseñadas a propósito para subrayar los efectos específicos del MDMA en comparación con otras drogas. Por ejemplo, los redobles del aerohouse y los intensos golpeteos de las cuerdas de guitarra aspiran 
a acentuar los efectos colectivos, extáticos y festivos en mucha mayor medida que los efectos hipnóticos o introspectivos"11. Esa conexión la conocen muy bien los creadores de ambientes en las discotecas españolas de las últimas dos décadas tal y como describe Oleaque ${ }^{15}$. Las discotecas españolas de mediados de los ochenta, especialmente de Ibiza ('Balearic beat'), se sitúan en el origen de formas musicales, tipos de discoteca, estilos de vida, formas de consumo de drogas, luego desarrolladas en Inglaterra por el movimiento house ${ }^{13}$.

La mayoría de las actuaciones preventivas en los ámbitos recreativos -poco usuales y más bien puntuales- adolecen frecuentemente de una base teórica y metodológica firme, presentan unos objetivos muy limitados, se basan principalmente en suministrar información a los jóvenes sobre las consecuencias del uso de drogas, testado de las pastillas o proponer actividades alternativas y no están evaluadas en sus resultados ${ }^{16,17}$. Existen orientaciones más dirigidas a modificar el contexto como club health, safer dance...., que tienen más posibilidades de mostrar alguna eficacia aunque también la evaluación es pobre ${ }^{16,18}$. Dentro de esta orientación tienen muy en cuenta la importancia en la prevención de problemas (embriagueces, peleas, primeros auxilios,...) de los profesionales de las discotecas por lo que proponen formarlos y de hecho en países como Inglaterra existe un amplio consenso en la necesidad de exigir esta formación.

Este estudio persigue precisamente conocer a estos profesionales que desde distintas perspectivas mantienen contacto con los jóvenes que salen a divertirse el fin de semana, pues de ellos depende en parte los determinantes ambientales (físicos y culturales) que crean y mantienen normas, expectativas, valores grupales e individuales y contextos favorables o no al consumo. Los objetivos específicos del estudio son conocer los consumos de drogas y opiniones sobre el consumo y la diversión en contextos recreativos de los profesionales que realizan una labor mediadora en el contexto recreativo y entornos juveniles e intentar explorar la relación que esto pueda tener en su relación con los jóvenes en materia de diversión y de consumo de drogas, cara a plantear colaboración preventiva a través de ellos.

\section{MATERIAL Y MÉTODO}

Para realizar el estudio empírico se ha realizado una encuesta a 246 profesionales repartidos en cinco Comunidades Autónomas (Baleares, Galicia, Madrid, País Vasco, Valencia). Además se han realizado un total de 20 entrevistas en profundidad a distintos profesionales.

Estos profesionales se han clasificado en 4 categorías que tienen contacto de una forma u otra con jóvenes que salen con frecuencia a divertirse las noches de los fines de semana, lo cual permite tener una visión del panorama existente, pero no agota todas las posibilidades de selección de la muestra. Estos grupos son:

Profesionales de la industria recreativa (61 profesionales, un 24,8\% del total de la muestra elegida), que están directamente relacionados con los locales y eventos recreativos creados para los jóvenes. Se trata de gerentes de locales, disk jockeys, relaciones públicas de discotecas, camareros, etc.

Profesionales de los medios de comunicación expresamente dirigida a los jóvenes (47 personas, 19,1\%), que dan información sobre estilos de vida, lideres juveniles, contribuyen a crear estilos de diversión, identidades, valores, a difundir los eventos más importantes de la ciudad, información sobre drogas,...

Profesionales de los servicios sociales y programas preventivos (109 personas, 44,3\%) en especial relación con los jóvenes que salen a divertirse. Se trata normalmente de trabajadores sociales o educadores que trabajan con jóvenes, algunos en espacios de ocio, otros haciendo prevención dentro del ámbito recreativo, en acciones como dar información sobre drogas, normalmente dentro de un programa de reducción del riesgo, que son los más frecuentes en nuestro país. Algunos de ellos trabajan con jóvenes en situación de riesgo o como animadores socioculturales.

Responsables de asociaciones juveniles (29 personas, $11,8 \%$ ) dedicadas a actividades de ocio y tiempo libre. Se ha creído que estos profesionales contribuyen a crear actividades de diversión distintas al 'salir de marcha' pero que también conocen, participan e interaccionan con esa forma de diversión.

Estos profesionales mediadores en espacios recreativos fueron encuestados por Irefrea - España en verano-otoño del año 2003, dentro de un estudio más amplio de ámbito europeo. Se utilizó un cuestionario estructurado que valoraba variables relativas a la formación, ámbito de actuación, vida recreativa y consumo de drogas de estos agentes sociales, junto con una serie de actitudes y percepciones relativas a la vida recreativa y consumo de drogas de los jóvenes con quienes interactuaban en su ámbito de actuación.

Los procedimientos estadísticos utilizados para el análisis de datos fueron: 1) Análisis descriptivos: Frecuencias, porcentajes y descriptivos básicos; 2) Tablas 
de contingencia con los estadísticos basados en el Chi cuadrado, calculados mediante pruebas exactas; 3 ) Análisis factoriales de componentes principales, aplicando la rotación equamax a los factores obtenidos; y 4) Análisis univariante de la varianza (ANOVA). Los análisis estadísticos fueron realizados con el paquete SPSS (versión 10.1).

\section{RESULTADOS}

\section{Datos sociodemográficos.}

El estudio sociodemográfico de los profesionales entrevistados permite constatar que se trata básicamente de gente joven. La mayoría, el 70\% son menores de 30 años, un 22\% tienen entre 31 y 40 años y sólo un $8 \%$ son mayores de 40 años. Es decir, que se ha entrevistado a unos profesionales para que den información sobre los jóvenes pero que ellos mismos son a su vez en su mayoría jóvenes. Ese hecho es de suma importancia metodológica porque la información recibida tiene un doble valor, los entrevistados definen a los 'otros', los usuarios con quienes trabajan, pero al tiempo están definiéndose a ellos mismos ya que definen a su propia generación a través de algunas de las cuestiones planteadas.

Los profesionales englobados en estos cuatro colectivos trabajan en distintas tareas o ámbitos expertos (tabla 1). El grupo mayoritario está formado por un $27 \%$ que son educadores de calle. Otros grupos importantes representados son los responsables de asociaciones juveniles $(9,3 \%)$, gerentes de locales recreativos $(9,3 \%)$ y trabajadores sociales $(8,5 \%)$. Hay que indicar que tanto la muestra en su conjunto como los porcentajes tanto por profesiones como en cada uno de los cuatro grupos no responde a criterios de representatividad estadística, sino a buscar un acercamiento a estos grupos para poder iniciar unas primeras reflexiones sobre cada uno de ellos. Se ha buscado, por descontado en cada una de las ciudades, que hubiese una representación amplia y variada de profesionales dentro de cada uno de los grupos.

Existe una ligera mayor representación de los varones (el 55,6\%) y la mayoría están solteros (el 64\%), mientras que el 35,7\% están casados o viven en pareja; muy pocos tienen hijos (solo el 16\%). Algo más de la mitad $(54,8 \%)$ definen la familia donde han nacido como de clase media alta y la otra mitad $(40,4 \%)$ de clase media baja. Hay un porcentaje considerable de universitarios (70\%), una cuarta parte tiene educación secundaria y solo un 7,7\% tiene una educación básica. Ante cuestiones religiosas son más bien poco creyentes (el 66,7\% consideran la religión poco o nada importante) y ante cuestiones políticas tienden
Tabla 1. Actividad de los profesionales en el espacio recreativo

\begin{tabular}{|c|c|}
\hline Educador de calle........ & $64(27,1 \%)$ \\
\hline $\begin{array}{l}\text { Líder / Responsable de una } \\
\text { asociación juvenil }\end{array}$ & $22(9,3 \%)$ \\
\hline $\begin{array}{l}\text { Gestor / propietario o gerente local } \\
\text { recreativo }\end{array}$ & $22(9,3 \%)$ \\
\hline Trabajador social & $20(8,5 \%)$ \\
\hline 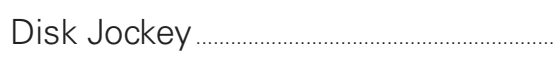 & $13(5,5 \%)$ \\
\hline Redactor en fancines o folletos...... & $\ldots .11(4,7 \%)$ \\
\hline Redactor en revistas juveniles ......... & $\ldots 10(4,2 \%)$ \\
\hline Locutor de radio & $\ldots .10(4,2 \%)$ \\
\hline Portero en una discoteca & $\ldots .10(4,2 \%)$ \\
\hline 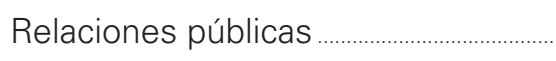 & $\ldots .5(2,1 \%)$ \\
\hline 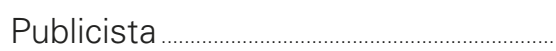 & $\ldots .5(2,1 \%)$ \\
\hline
\end{tabular}

Otros (actores, camareros, monitores, músicos, etc.) $44(18,6 \%)$

bastante hacia el centro izquierda (más concretamente el $31,4 \%$ se autodefine de centro, de izquierda un $49 \%$, de extrema izquierda un $14,7 \%$ y sólo un $4,9 \%$ se define de centro derecha).

Obviamente salir de marcha es una actividad en la que participan los mediadores en su tiempo libre tanto por edad, como porque les gusta; una tercera parte $(33,3 \%)$ sale de tres a cuatro fines de semana al mes, otra tercera parte $(36,6 \%)$ uno o dos fines de semana al mes y la tercera $(30,1 \%)$ parte que queda menos de una vez al mes.

La música es un medio de socialización de los jóvenes ${ }^{19}$ y que les interesa tanto a ellos como a los mediadores. Los mediadores entrevistados se adscriben en primer lugar a la música Rock (el 42,7\%), un $21,3 \%$ se adscribe al pop y algunos menos, el $14,2 \%$, al dance, house...

\section{Consumo de drogas.}

El consumo de drogas de los mediadores entrevistados consideramos que es un dato importante, no tanto en cuanto a lo que se refiere a su conducta privada, sino por la posibilidad de influir en su relación con los jóvenes y en su visión de los problemas relacionados con las drogas. Se les ha preguntado si al menos de vez en cuando se embriagan, consumen cannabis o alguna otra droga ilegal. La mayoría (152, $62 \%)$ de estos mediadores se embriaga de vez en cuando, el 38,5\% (94) son consumidores de cannabis al menos ocasionalmente y el $25 \%$ (61) consumen al menos de vez en cuando otras drogas ilegales. 
El consumo de drogas es distinto según el colectivo. Los miembros de los servicios sociales y de las asociaciones juveniles se embriagan, consumen cannabis y otras drogas en un porcentaje menor que los agentes de la industria recreativa y de los medios de comunicación, pero aún así se trata siempre de porcentajes importantes (ver tabla 2)
Es de interés observar que la opinión que se tiene sobre las drogas depende en algunos casos del propio consume que se hace, especialmente en cómo se percibe el riesgo asociado al consumo de cannabis. Por ejemplo, la opinión sobre la peligrosidad del consumo frecuente de marihuana varia según se es o no consumidor excesivo de alcohol, o de si se consume o

\begin{tabular}{|lccccc|}
\hline \multicolumn{5}{|c|}{ Tabla 2. Consumo de drogas según colectivos de profesionales } \\
\hline & Total (n=246) & Servicios sociales & Asociación juvenil & Industria & Medios \\
\hline Se embriaga & $152(62 \%)$ & $61(56,5 \%)$ & $18(62,1 \%)$ & $38(62,3 \%)$ & $35(74,5 \%)$ \\
Consume cannabis & $94(38,4 \%)$ & $38(35,2 \%)$ & $8(27,6 \%)$ & $27(44,3 \%)$ & $21(44,7 \%)$ \\
Consume otras drogas & $61(25 \%)$ & $20(18,5 \%)$ & $4(14,3 \%)$ & $24(39,3 \%)$ & $13(27,7 \%)$ \\
\hline
\end{tabular}

\section{Percepción del riesgo.}

La percepción del riesgo de los mediadores respecto al consumo de drogas es, paradójicamente, relativamente muy alta cuando se trata de enjuiciar (ver tabla 3) una serie de pautas de consumo de drogas legales e ilegales ¿Cómo es que si perciben estos peligros consumen ellos mismo con tanta frecuencia? La causa de esta disonancia no la podemos concretar en función de nuestros datos.

Destacamos también como el consumo del cannabis preocupa menos que el consumo de tabaco, opinión también frecuente entre los jóvenes y en consonancia con el estado actual de la calidad de la información sobre el cannabis que circula en los medios de comunicación social.

Solamente se encuentra una asociación estadísticamente significativa entre la peligrosidad percibida en la embriaguez cada fin de semana y el colectivo; los profesionales de los medios tienen una probabilidad mayor de lo esperado de considerar ese abuso de alcohol como poco o nada peligroso $\left(\mathrm{Chi}^{2}=9,9\right.$; $\mathrm{p}$ exacto de Monte Carlo < 0,05 para 3 grados de libertad). no cannabis u otras drogas ilegales (ver tabla 4). Tanto los que abusan de alcohol, como los que consumen cannabis $u$ otras drogas ilegales tienden a ver menos problemático el uso de dicha sustancia. Sin embargo, la percepción de peligro en el consumo habitual de alcohol en exceso, tabaco, cocaína o éxtasis no se relaciona sustancialmente con el hecho de que los mediadores consuman alcohol, cannabis u otras drogas.

En cuanto a las asociaciones entre el consumo de drogas de los mediadores y su percepción del riesgo en el consumo de drogas:

1. Los mediadores que consumen alcohol hasta la embriaguez perciben un riesgo significativamente menor que los que no lo hacen, tanto en el uso regular de éxtasis $\left(\mathrm{Chi}^{2}=6,7 ; \mathrm{p}\right.$ exacto de Monte Carlo < 0,05 para 1 grado de libertad) como en el consumo habitual de cannabis $\left(\mathrm{Chi}^{2}\right.$ = 5,3; p exacto de Monte Carlo < 0,05 para 1 grado de libertad).

2. Los mediadores que consumen cannabis perciben un riesgo significativamente menor que los que no lo hacen en el consumo habitual de can-

\begin{tabular}{|lccccc|}
\hline \multicolumn{5}{|c|}{ Tabla 3. Percepción del riesgo asociado al consumo: } \\
Conductas que consideran peligrosas o muy peligrosas \\
\hline & Total & $\begin{array}{c}\text { Servicios } \\
\text { sociales }\end{array}$ & $\begin{array}{c}\text { Asociación } \\
\text { juvenil }\end{array}$ & Industria & Medios \\
\hline Consumir cocaína cada fin de semana & $242(99,6 \%)$ & $107(100 \%)$ & $29(100 \%)$ & $60(100 \%)$ & $46(97,9 \%)$ \\
Tomar éxtasis cada fin de semana & $239(98,4 \%)$ & $106(99,1 \%)$ & $29(100 \%)$ & $58(96,7 \%)$ & $46(97,9 \%)$ \\
Embriagarse cada fin de semana & $218(89,7 \%)$ & $102(95,3 \%)$ & $26(89,7 \%)$ & $53(88,3 \%)$ & $37(78,7 \%)$ \\
Fumar un paquete de cigarrillos al día & $221(90,9 \%)$ & $96(89,7 \%)$ & $28(96,6 \%)$ & $56(93,3 \%)$ & $41(87,2 \%)$ \\
Fumar marihuana con regularidad & $170(70,2 \%)$ & $79(74,5 \%)$ & $24(82,8 \%)$ & $36(60 \%)$ & $31(66 \%)$ \\
\hline
\end{tabular}


Tabla 4. Diferencias entre los que están de acuerdo con la siguiente afirmación según si se emborrachan, consumen cannabis $u$ otras drogas

\begin{tabular}{|lcccccc|}
\hline & \multicolumn{2}{c}{ Se emborrachan } & Consume cannabis & \multicolumn{2}{c|}{$\begin{array}{l}\text { Consume otras } \\
\text { drogas ilegales }\end{array}$} \\
\cline { 2 - 7 } & Si (\%) & No (\%) & Si (\%) & No (\%) & Si (\%) & No (\%) \\
\hline $\begin{array}{l}\text { Fumar marihuana regularmente } \\
\text { es peligroso o muy peligroso }\end{array}$ & $98(64,9)$ & $71(78,9)$ & $46(49,5)$ & $123(83,1)$ & $28(45,9)$ & $140(78,2)$ \\
$\begin{array}{l}\text { Fumar un paquete de cigarrillos } \\
\text { al día es peligroso o } \\
\text { muy peligroso }\end{array}$ & $135(89,4)$ & $85(93,4)$ & $85(90,4)$ & $135(91,2)$ & $57(93,4)$ & $162(90)$ \\
$\begin{array}{l}\text { Embriagarse cada fin de semana } \\
\text { es peligroso o muy peligroso }\end{array}$ & $133(88,1)$ & $84(92,3)$ & $84(89,4)$ & $133(89,9)$ & $53(86,9)$ & $163(90,6)$ \\
$\begin{array}{l}\text { Consumir cocaína cada fin de } \\
\text { semana es peligroso } \\
\text { o muy peligroso }\end{array}$ & $191(100)$ & $90(98,9)$ & $94(100)$ & $147(99,3)$ & $61(100)$ & $179(99,4)$ \\
$\begin{array}{l}\text { Tomar éxtasis cada fin de } \\
\text { semana es peligroso o } \\
\text { muy peligroso }\end{array}$ & $151(100)$ & $87(95,6)$ & $92(97,9)$ & $146(98,6)$ & $59(96,7)$ & $178(98,9)$ \\
\hline
\end{tabular}

nabis $\left(\mathrm{Chi}^{2}=30,9\right.$; $\mathrm{p}$ exacto de Monte Carlo < 0,001 para 1 grado de libertad).

3. Lo mismo ocurre con los mediadores que consumen alguna otra droga ilegal y su percepción del riesgo implícito en el uso regular de cannabis, al ser comparados con los que no consumen $\left(\mathrm{Chi}^{2}=22,6\right.$; $\mathrm{p}$ exacto de Monte Carlo $<0,001$ para 1 grado de libertad).

\section{Percepción de los jóvenes que salen de marcha.}

Los jóvenes vistos a través de los ojos de los mediadores aparecen descritos como relativamente poco motivados hacia metas vitales importantes y como relativamente más materialistas que idealistas. A partir de una lista de posibles intereses de los jóvenes (ver tabla 5), una mayoría (un 54,9\%) piensa que a los jóvenes les motiva bastante tener dinero y encontrar un trabajo (un 41,6\%). En el otro extremo encontraríamos que solo algunos jóvenes tienen claro interés en 'entenderse con los adultos' (un 7,4\%) o en actuar sobre el uso de drogas (un 5,7\%). En una posición intermedia $(23,4 \%)$ están los mediadores que piensan que para los jóvenes es importante independizarse de sus familias.

¿Qué opinan sobre la diversión de los jóvenes?. Una mayoría $(66,5 \%)$ de los mediadores considera que los jóvenes actuales son unos privilegiados por el tiempo libre del que disponen, al mismo tiempo que piensan $(60 \%)$ que salir de marcha es muy bueno porque los jóvenes deberían divertirse todo lo que puedan o $(51 \%)$ que gastan todo su dinero en salir de marcha. También piensan $(48,3 \%)$ que los jóvenes tienen claro qué hacer para divertirse.

Dos de las anteriores cuestiones presentan diferencias sustanciales o significativas entre colectivos (ver tabla 6). Los que trabajan para los Servicios sociales y las asociaciones juveniles tienen menos claro que salir de marcha sea muy bueno (las diferencias de los primeros con el resto son estadísticamente significativas en este ítem: $\left(\mathrm{Chi}^{2}=12,6\right.$; p exacto de Monte Carlo $<0,01$ para 3 grados de libertad)) y creen además que los jóvenes no tienen tan claro la forma en que tienen que divertirse.

El $66,7 \%$ del conjunto de mediadores considera que si salen tanto de marcha es porque no conocen otra forma de divertirse. También opinan $(43,1 \%)$ que es la actividad más divertida que pueden hacer. Es decir, parece que en el panorama actual de la diversión salir de marcha se ha convertido en casi la única opción según su opinión mayoritaria.

El propio consumo de drogas entre los mediadores parece que también puede determinar (ver tabla 7) su visión sobre la claridad de ideas de los jóvenes acerca de su diversión y acerca de la supuesta bondad y beneficios de salir de marcha. 
Tabla 5. Consideran que los jóvenes son bastante o muy dinámicos en

lograr los siguientes objetivos (\%)

Tener dinero

$134(54,9)$

Encontrar un trabajo

$102(41,6)$

Prepararse como profesionales

$78(31,8)$

Actuar contra el SIDA

$61(24,9)$

Llegar a ser independientes de su familia

$57(23,4)$

Ahorrar para comprarse una vivienda

$40(16,3)$

Llevar un estilo de vida saludable

$38(15,5)$

Tener pareja estable

$26(10,7)$

Entenderse mutuamente con los adultos

$18(7,4)$

Actuar contra el uso de drogas

$14(5,7)$

\section{Tabla 6. Están de acuerdo ante las siguientes afirmaciones respecto a los jóvenes y la diversión por colectivos}

\begin{tabular}{|lcccc|}
\hline & Servicios sociales & Asociaciones juveniles & Industria & Media \\
\hline $\begin{array}{l}\text { Los jóvenes tienen claro } \\
\text { qué hacer para divertirse }\end{array}$ & $37(34,3 \%)$ & $12(42,9 \%)$ & $36(60 \%)$ & $32(69,6 \%)$ \\
\hline $\begin{array}{l}\text { Salir de marcha es } \\
\text { muy bueno }\end{array}$ & $55(50,9 \%)$ & $13(48,1 \%)$ & $46(75,4 \%)$ & $32(68,1 \%)$ \\
\hline
\end{tabular}

Tabla 7. Diferencias entre los que están de acuerdo con la siguiente afirmación respecto a los jóvenes y la diversión según si se emborrachan, consumen cannabis u otras drogas

\begin{tabular}{|lcccccc|}
\hline & \multicolumn{2}{c}{ Se emborrachan } & \multicolumn{2}{c|}{ Consume cannabis } & \multicolumn{2}{c|}{ Consume otras drogas ilegales } \\
\cline { 2 - 6 } & Si (\%) & No (\%) & Si (\%) & No (\%) & Si (\%) & No (\%) \\
\hline $\begin{array}{l}\text { Los jóvenes tienen claro } \\
\text { qué hacer para divertirse }\end{array}$ & $73(49,3)$ & $43(46,3)$ & $43(46,7)$ & $73(49)$ & $31(52,5)$ & $84(46,4)$ \\
$\begin{array}{l}\text { Salir de marcha es } \\
\text { muy bueno }\end{array}$ & $91(61)$ & $53(58,3)$ & $90(64,5)$ & $85(57)$ & $41(67,2)$ & $103(57,2)$ \\
\hline
\end{tabular}

En cuanto a la claridad de ideas de los jóvenes para divertirse, los mediadores que consumen otras drogas ilegales enfatizan ligera y significativamente más esa claridad en los jóvenes $\left(\mathrm{Chi}^{2}=7,9 ;\right.$ p exacta $<0,05$ para 3 grados de libertad). La presumible bondad de salir de marcha es más enfatizada por los consumidores, aunque las diferencias no son estadísticamente significativas. La diferencia con los no-consumidores es mayor en el consumo de cannabis que en el consumo de alcohol, y aún es mayor en las demás drogas ilegales que en el cannabis.

\section{Creencias y mitos sobre las drogas.}

Las creencias y mitos de los mediadores sobre las drogas son también muy importantes pues nos informarán de su nivel de información y acerca de hasta que punto se diferencian sus creencias de las creencias que circulan normalmente entre los jóvenes. El consumo de drogas se legitima a través de una serie de ideas y mitos acerca de su valor instrumental para el logro de diversas metas, y acerca de los riesgos y consecuencias que se derivan de su consumo. Nos interesa saber qué piensan estos colectivos de pro- 
fesionales pues ello condicionará seguramente los mensajes y actuaciones que tienen con los jóvenes con los que están en contacto. Por poner un ejemplo de lo que queremos decir, la mitad de ellos $(50,5 \%)$ opina que tomar drogas es una forma de rebelión, lo cual, además de no ser ajustado a la realidad -por ej., según la Encuesta Escolar 2002 del PND sólo un $2,3 \%$ de los escolares piensan que el hecho de que esté prohibido puede suponer un aliciente, mientras que las motivaciones que predominan para el consumo de drogas son de carácter lúdico: la diversión, la experimentación de nuevas sensaciones, el placer-, puede inducir a legitimar su consumo, es decir, a dar un sentido positivo al consumo.

Esta y otras creencias o mitos tienen una aceptación distinta según el colectivo de mediadores que consideremos. En la tabla 8 se muestran las diferencias ante dos de esas creencias. Vemos claramente cómo los colectivos de profesionales de la industria y de los medios de comunicación tienen con mayor frecuencia una percepción del cannabis como sustancia no perjudicial, al igual que creen que la dieta y el ejercicio pueden compensar los problemas de las drogas (las diferencias estadísticamente significativas se encuentran entre los profesionales de la industria y los demás en este segundo ítem).

Pero lo que posiblemente más marca la diferencia ante la justificación de los mitos legitimadores del consumo es el consumo que hacen de algunas drogas los propios mediadores. Se puede ver muy claramente (ver tabla 9) de que forma los que abusan del alcohol (llegan a embriagarse), los que consumen cannabis o los que consumen otras drogas ilegales al menos de vez en cuando tienen opiniones mucho más favorables y legitimadoras del consumo de drogas que los que no consumen.

\section{Motivaciones para el consumo.}

En la tabla 10 se continúa indagando acerca de sus opiniones sobre lo que motiva el consumo. El 91,8\% afirma que la facilidad para conseguir drogas es lo que explica que las drogas sean tan populares entre los jóvenes. Esa es la razón más valorada para el colectivo de profesionales entrevistados. Los datos que

Tabla 8. Algunos mitos según colectivos

\begin{tabular}{|lccccc|}
\hline & $\begin{array}{c}\text { Servicios } \\
\text { sociales }\end{array}$ & $\begin{array}{c}\text { Asociación } \\
\text { juvenil }\end{array}$ & Industria & Medios & Total \\
\hline $\begin{array}{l}\text { Los efectos negativos de las drogas } \\
\text { pueden ser compensados con una } \\
\text { dieta adecuada y ejercicio }\end{array}$ & $29(27,6 \%)$ & $9(31 \%)$ & $38(63,3 \%)$ & $19(40,0 \%)$ & $95(39,4 \%)$ \\
\hline $\begin{array}{l}\text { El cannabis no es una sustancia } \\
\text { perjudicial }\end{array}$ & $31(29,5 \%)$ & $7(25 \%)$ & $31(51,7 \%)$ & $23(48,9 \%)$ & $92(38,4 \%)$ \\
\hline
\end{tabular}

Tabla 9. Diferencias entre los que están de acuerdo con algunos mitos según si se emborrachan, consumen cannabis $u$ otras drogas

\begin{tabular}{|lcccccc|}
\hline & \multicolumn{3}{c}{ Se embriagan } & Consume cannabis & $\begin{array}{c}\text { Consume otras } \\
\text { drogas ilegales }\end{array}$ \\
\cline { 2 - 7 } & Si (\%) & No (\%) & Si (\%) & No (\%) & Si (\%) & No (\%) \\
\hline $\begin{array}{l}\text { Algunas personas exageran los } \\
\text { problemas asociados al consumo } \\
\text { de drogas }\end{array}$ & $85(55,9$ & $28(31,8)$ & $58(63)$ & $55(37,2)$ & $44(72,1)$ & $68(38,2)$ \\
$\begin{array}{l}\text { El peligro no está en las drogas } \\
\text { sino en su adulteración }\end{array}$ & $100(65,8)$ & $34(38,6)$ & $62(67,4)$ & $72(48,6)$ & $46(75,4)$ & $87(48,9)$ \\
$\begin{array}{l}\text { El cannabis no es una sustancia } \\
\text { perjudicial }\end{array}$ & $72(47,7)$ & $20(22,7)$ & $53(58,2)$ & $39(26,4)$ & $40(65,6)$ & $51(28,8)$ \\
$\begin{array}{l}\text { La vida recreativa sin drogas no } \\
\text { es divertida }\end{array}$ & $18(11,9)$ & $4(4,5)$ & $17(18,7)$ & $5(2,3)$ & $13(14,8)$ & $9(5,1)$ \\
\hline
\end{tabular}


Tabla 10. Por qué las drogas se han convertido en tan populares (\%)

Se consiguen fácilmente

$225(91,8)$

Las drogas están promocionadas por los medios de comunicación

(películas, televisión, publicidad, canciones de moda, etc.)

$193(79,1)$

Porque la gente no es consciente de los problemas que causan

$183(75,3)$

Porque hay carencia de información acerca de sus consecuencias negativas

$136(55,8)$

La conexión entre quienes consumen las mismas sustancias es muy agradable

$123(51,1)$

Son tan buenas que no necesitan promoción

$70(28,6)$

Sin drogas no tiene sentido salir de marcha

$29(11,9)$

aportan los dos eurobarómetros de 2002 y 2004 20:21 también confirman que la accesibilidad de las drogas es un hecho generalizado, en especial en el contexto recreativo (así lo afirma más del 90\% de los jóvenes españoles que han participado en dicho estudio que sitúa a los jóvenes españoles entre los que afirman tener más fácil acceso a los drogas ilegales en toda Europa).

Al realizar el análisis factorial de componentes principales para agrupar doce ítems relativos a las drogas y los motivos de su popularidad, la mayoría de estos se agrupan en tres factores en una solución que explica casi la mitad de su varianza (45\%). La tabla 11 representa los dos factores más sustanciales y los 9 ítems con mayor representación en alguno de estos dos factores interpretados.

Estos dos factores podrían interpretarse del siguiente modo:

1. El factor denominado "Riesgo asumible", consistente en entender el uso de drogas como algo útil para la diversión, que favorece la interacción entre quienes las consumen, y que en todo caso es un riesgo más, propio de la diversión normalizada y, por consiguiente, asumible.

2. El factor denominado "Baja peligrosidad", que agrupa la percepción de inocuidad y ausencia de potencial adictivo de las drogas en general (el peligro está en la adulteración) y del cannabis en particular, y cuyos "peligros" son exagerados por algunas personas.

Nuevamente se observa que el ámbito de actuación también se asocia sustancial y significativamente con el concepto de baja peligrosidad de las drogas (ANOVA, $F=6,2 ; 3$ grados de libertad; $p<0,001$ ). Quienes puntúan más alto son los mediadores en la industria recreativa, seguidos de los profesionales en medios de comunicación. En el polo opuesto se sitúan los mediadores en el mundo asociativo, que perciben una peligrosidad casi tan elevada como los mediadores en servicios sociales. Los mediadores en los servicios sociales puntúan significativamente más bajo que los mediadores en la industria recreativa en esta percepción de inocuidad de las drogas.

Si comparamos a los mediadores que se emborrachan con los que no lo hacen, y los que consumen

Tabla 11. Análisis factorial sobre motivos acerca de la popularidad de las drogas: cargas factoriales de 9 ítems en los dos primeros factores resultantes.

\begin{tabular}{|lc|}
\hline & \multicolumn{2}{c|}{ Componentes } \\
\cline { 2 - 2 } & $\mathbf{1}$ \\
\hline Sin drogas no tiene sentido salir de marcha &, 69 \\
La conexión entre consumidores es muy agradable &, 66 \\
Son tan buenas que se promocionan solas & 64 \\
La vida recreativa sin drogas no es divertida &, 63 \\
Las drogas son un riesgo más, la vida sin riesgo no merece la pena &, 47 \\
El cannabis no es una sustancia perjudicial &, 79 \\
El peligro está en la adulteración, no en las drogas &, 72 \\
Solo la heroína es realmente adictiva &, 54 \\
Se exageran los problemas asociados a las drogas &, 51 \\
\hline
\end{tabular}


cannabis u otras drogas ilegales con los que no consumen, los resultados de estas tres comparaciones siempre indican una puntuación significativamente más baja en la peligrosidad de las drogas entre los consumidores ( $p<0,001$ en los tres contrastes basados en el análisis de varianza). Además, estos tres efectos son estadísticamente independientes del colectivo profesional que consideremos. Los consumidores de alcohol en exceso, cannabis u otras drogas ilegales justifican significativamente más que los que no consumen el uso de drogas como un 'riesgo asumible', aunque las diferencias son menos acentuadas que en la percepción de baja peligrosidad ( $p<0,05$ en los dos contrastes basados en el análisis de varianza para el uso de alcohol y cannabis y $p<0,001$ para el uso de alguna otra droga ilegal).

\section{DISCUSIÓN}

Se ha presentado un acercamiento a las consecuencias que puede tener el uso de drogas en cuatro colectivos de profesionales o mediadores sociales que están en contacto inmediato de una u otra forma con los jóvenes que suelen salir a divertirse los fines de semana. Aunque no se trata de muestras significativas, si son muestras relativamente amplias y bien seleccionadas como para poder establecer un acercamiento a estos colectivos y poder abrir un debate sobre cuestiones claves para la prevención en estos ámbitos.

Estos grupos de profesionales son básicamente un colectivo joven, que tiene entre sus formas de divertirse el salir de marcha y que hace un consumo relativamente importante de alcohol, cannabis y otras drogas ilegales. Son bastante preocupantes todas las cifras de consumo, pero especialmente, por su significación, las del colectivo de profesionales que trabajan en prevención o en servicios sociales en contacto directo con los jóvenes que salen de marcha. Recordemos que de este último colectivo un $56,5 \%$ se embriagan con alguna frecuencia (muy por encima del 21,2\% durante el último año encontrado en población general española en la encuesta domiciliaria del 200322), un $35,2 \%$ fuman cannabis (muy por encima del 11,3\% de consumidores durante el último año encontrado en población general española en la encuesta domiciliaria del 2003) y un $18,5 \%$ usan otras drogas ilegales. Si bien es verdad que estas cifras de consumo son menores que las de los colectivos de profesionales que trabajan en la industria o en los medios de comunicación juveniles y que frente a diversas cuestiones (por ejemplo si el cannabis es perjudicial) este grupo de profesionales tiene un posicionamiento más cercano a las necesidades preventivas, no dejan de estar lejanos de las pretendidamente ideales de profesionales supuestamente formados que tienen que realizar una tarea con jóvenes que precisamente tienen o pueden tener problemas con el consumo de diversas sustancias. Obviamente esto no tendría relevancia si el consumo privado no influyese en su percepción de los problemas de los jóvenes y, posiblemente, en su relación con ellos, como de hecho sí ocurre.

La percepción de los riesgos es un constructo central en la prevención en general y en el consumo de drogas en particular. Sabemos que la alta percepción de riesgo se asocia a un menor consumo ${ }^{23}$. En el presente estudio también se encuentra el mismo fenómeno: los que se emborrachan o consumen cannabis $u$ otras drogas tienden a ver menos problemas asociados al consumo. Por ejemplo entre los que consumen cannabis un $58 \%$ creen que el cannabis no es perjudicial y si hablamos de los que no lo consumen nos encontramos con que solamente es un $26 \%$ los que lo ven de esta forma.

Las creencias y mitos sobre las drogas pueden ejercer una función favorable o desfavorable a la legitimación, promoción o tolerancia del consumo de drogas. Las opiniones de estos colectivos estudiados son relevantes pues - lo sepan ellos mismos o no- ejercen una función mediadora entre las necesidades de la sociedad y los jóvenes. Tenemos en este sentido que un $38,4 \%$ de toda la muestra creen que el cannabis no es perjudicial, estando más cerca pues de los mitos de los propios jóvenes que lo que sería deseable para profesionales que ocupan lugares clave en la socialización de los jóvenes, que de la evidencia científica ${ }^{24 ; 25}$. Estaríamos por consiguiente en una situación en que un grupo importante de profesionales que están muy cercanos a los jóvenes tienen dificultades para tomar distancia entre lo que podría ser su mundo privado en relación a lo que concierne a las necesidades profesionales -entendidas por lo menos desde el punto de vista de la prevención y de la socialización de los jóvenes- viniendo ello agravado de forma decisiva por sus propios consumos. El intento de dar congruencia al propio consumo de drogas por parte de los consumidores acaba dificultando el papel profesional en relación con estas áreas de consumo de drogas que estamos analizando, lo cual es especialmente preocupante sobre todo si se trata de profesionales de los servicios sociales, de la prevención o del mundo asociativo juvenil. También tiene su importancia los altos consumos de los profesionales de la industria y de los medios de comunicación, pero ello tendría una más fácil explicación pues existe una escasísima conciencia dentro de este colectivo acerca de que tienen responsabilidades sobre la socialización de los jóvenes y además es la propia industria quien los contrata.

Nuestros resultados indican la necesidad de revisar y actualizar los planteamientos de estos mediadores 
en materia del consumo de drogas y la problemática de los jóvenes, de modo que responda más a los actuales conocimientos y a las metas que tiene la comunidad que a intereses personales. También inciden nuestros resultados en la necesidad de que los responsables de políticas juveniles y programas de prevención relacionados con el ocio se replanteen sus objetivos y prioricen la formación de los mediadores.

\section{AGRADECIMIENTOS}

Este estudio se ha realizado con la ayuda de una subvención de la Delegación del Gobierno para el Plan Nacional sobre Drogas.

\section{REFERENCIAS}

1. Plan Nacional sobre Drogas. Observatorio Español sobre drogas. Informe $n^{\circ}$ 5. Madrid: Plan Nacional sobre Drogas, 2002.

2. Calafat $A$, Juan $M$, Becoña $E$, Fernández C, Gil E, Llopis JJ. Organización y estrategias de la cultura pro cannabis. Adicciones 2000;12 (supl 2):231-74.

3. Shields D, Carol J, Balbach E. Hollywood on tobacco: how the entertainment industry understand tobacco portray. Tobacco Control 1999; 378-86.

4. Kemmesies U. Use of illicit drugs in the 'civil' milieu: on the influence of formal and informal social control -preliminary finding of a pilot study-. Originalbeiten 2000; 46: 101-10.

5. Rhodes $T$, Lilly R, Fernández $C$ et al. Risk factors associated with drug use: the importance of "risk environment". Drugs: education, prevention and policy 2003; 10:303-29.

6. Gifford R, Hine DW. Substance misuse and the physical environment: the early action on a newly completed field. Int J Addict 1990; 25 (7A-8A): 827-53.

7. Measham F, Parker $H$, Aldridge J. The teenage transition: from adolescent recreational drug use to the young adult dance culture in Britain in the mid-1990s. J Drug Issues 1998; 28:9-32.

8. Parker H, Aldridge J, Measham F. Illegal Leisure. The normalization of adolescent recreational drug use. London: Routledge, 1998.

9. Bellis MA, Hale G, Bennet LA, Chaves M, Kilfoyle M. Ibiza uncovered: changes in substance use and sexual behaviour amongst young people visiting an international night-life resort. Int J Drug Policy 2000; 11:235-44.

10. Calafat $A$, Fernández $C$, Juan M, Bellis MA, et al. Risk and control in the recreational drug culture. Palma de Mallorca: Irefrea, 2001.

11. Gilbert J, Pearson E. Cultura y políticas de la música dance. Disco, hip-hop, house, techno, drum'n'bass y garage. Barcelona: Paidós, 2003.

12 Thornton S. Club Cultures, Music, media and subcultural capital. Cambridge: Polito Press, 1995.

13 Collin M. Altered State: The Story of Ecstasy Culture and Acid House. London: Serpent's Tail, 1997.

14. Elzo J, et al. Las culturas de las drogas en los jóvenes. Ritos y fiestas. San Sebastian: Gobierno Vasco. Departamento de Justicia, trabajo y seguridad social, 2000.

15. Oleaque JM. En Ėxtasi. Drogues, música màkina i ball: viatge a les estranyes de <la festa>. Barcelona: Ara LLibres, 2004.

16. Calafat $A$, Fernández $C$, Juan $M$ et al. Enjoying the nightlife in Europe. The role of moderation. Palma de Mallorca: Irefrea, 2003.

17. Burkhart G and López M. Party Setting Projects from EDDRA. http://eddrapdf.emcdda.org/eddra_party_ settings.pdf. (10/10/03) . 2002.

18. Calafat A, Juan M. De la etiología a la prevención del uso y abuso de drogas recreativas. Adicciones 2003;15 (supl 2):261-88.

19. Green AM. Des Jeunes et des Musiques. París: L'Harmattan, 1997.

20. EOS Gallup Europe. Young people and drugs. Flash Eurobarometer 158. http://europa.eu.int/comm/public_ opinion/flash/fl158_en.pdf. (20/01/05) . 2004. European Commission.

21. The European Opinion Research Group. Attitudes and Opinions of young people in the European Union on Drugs. http://www.emcdda.eu.int/multimedia/project_ reports/policy_law/eurobarometer_youth_drugs.pdf. (Consultado 20/01/05) Eurobarometer 57.2, 1-46. 2002. European Commission.

22. Plan Nacional sobre Drogas. Encuesta domiciliaria sobre abuso de drogas en España 2003. http://www.msc. es/pnd/observa/pdf/domiciliaria2003.pdf. (10/12/04). 2004.

23. Johnston LD, O'Malley PM. Explaining recent increases in student' marijuana use: impact of perceived risks and disapproval, 1976 through 1996. Am J Public Health 1998;887-92.

24. Quiroga M. Cannabis: efectos nocivos sobre la salud mental. Adicciones 2000;12(supl 2):135-48.

25. Quiroga M. Cannabis: efectos nocivos sobre la salud física. Adicciones 2000;12(supl 2), 117-134. 
